\begin{tabular}{ccc}
\hline & International Journal of Engineering \& Technology, 7 (2.7) (2018) 878-882 \\
SPC & Website: www.sciencepubco.com/index.php/IJET \\
Research Paper & Technology \\
\hline
\end{tabular}

\title{
Time Frequency analysis of Non-Stationary signals by Differential frequency window $\mathrm{S}$-Transform
}

\author{
B. Murali Krishna, M Srinivas, S. Raja Gopal, G. L. P. Ashok \\ Department of Electronics and Communication Engineering, Koneru Lakshmaiah Education Foundation, \\ Guntur, Andhra Pradesh, Pin-522502. \\ *Corresponding author E-mail: murali.ece713@gmail.com
}

\begin{abstract}
The S transform is an extension of Short Time Fourier Transform and Wavelet transform, has a time frequency resolution which is far from ideal. A differential frequency window is proposed in this paper to enhance the time frequency energy localization. When a non stationary signal consists of abrupt amplitude variation equal to peak of Gaussian function at initial intervals of chosen guassian window, then some part of the signal amplitude will be nullified during transform projection. The major function of differential frequency window is to track all abrupt amplitude-frequency variations which exploits in non - stationary signals. A mathematical method namely Newton Raphson method is adopted for this trace. The proposed scheme is tested for ECG data in presence of noise environment and results shows that proposed algorithm produces better enhanced energy localization in comparison to the standard S - Transform, STFT, and CWT. Furthermore the above algorithm is implemented on FPGA for real time applications.
\end{abstract}

Keywords: Newton Raphson method, S-Transform, Localization, ECG.

\section{Introduction}

Analysis of non stationary signal acquires the highest priority in many of real time applications [1]. Several time frequency transforms are proposed earlier in which S- Transform is most popular technique used in signal analysis [2] [3] [4]. The STransform (ST), introduced by Stockwell et al.[5] is advanced method to short time Fourier transform(STFT) and continues wavelet transform(CWT) for progressive time frequency resolution [6] [7]. The invariable resolution of STFT and insufficient of phase data in CWT results in development of ST [8] [9]. ST provides cumulative resolution property of CWT with globally referenced phase output property [9]. During this progressive resolution energy concentration is major important factor for signal analysis [10]. Today in many of real time applications power spectral densities of signal are major requirement for signal analysis [11]. In such cases if the signal is contaminated by abrupt signal, ST suffers to provide time frequency analysis at faster rate due to lesser higher frequency localization [12] [13]. The following proposed method resolves spectral density components at higher rate with accuracy resolution. This paper is organized as follows in section 2 gives complete details about analytical approach for how window includes nonlinearity of non stationary signal. Section 3 implements the physical method for proposed algorithm. Section 4 includes simulation results and discussions. Finally Section 5 contains conclusion and limitations.

\section{Analytical approach identity}

Stock well transform of a signal $\mathrm{x}(\mathrm{t})$ is given by

$\mathrm{S}(\mathrm{t}, \mathrm{f})=\int_{-\infty}^{\infty} x(\tau) w(t-\tau, f) e^{\frac{-(t-\tau)^{2}}{2 \sigma(f)^{2}}} \mathrm{~d} \tau ;$

Where $\quad w(t-\tau, f)=\frac{1}{\sigma(f) \sqrt{2 \pi}} e^{\frac{-(t-\tau)^{2}}{2 \sigma(f)^{2}}}$;
Standard deviation $\quad \sigma(f)=\frac{1}{|f|}$

In this section an improved localization operators ( $t, f)$ are derived from the standard S-Transform with usual notations stated in [8,9 and 14].

Let window function $\mathrm{w} € \mathrm{R}$ such that

$\|w\|_{(R)}=1$.

And $\quad \int_{-\infty}^{\infty} \frac{\left|w^{\prime}(\xi-1)^{2}\right|}{\xi} \mathrm{d} \xi<\infty$;

By defining $1<\mathrm{S}<\infty$, the localization operator $S_{\sigma, W}: \stackrel{\text { yields }}{\longrightarrow} R$ given as

$\left(S_{\sigma, W}, t, f\right)=\frac{1}{c_{w}} \int_{-\infty}^{\infty} \int_{-\infty}^{\infty} \sigma(b, \xi)$

$\left(S_{W} t\right)(b, \xi) \overline{\left(S_{W} f\right)(b, \xi)} e^{\frac{-(t-b)^{2}}{2 \sigma^{2}}} e^{\frac{-(f-\xi)^{2}}{2 \sigma^{2}}} d b d \xi$; $\left(S_{\sigma, W}, t, f\right)=\frac{1}{c_{w}} \int_{-\infty}^{\infty} \int_{-\infty}^{\infty} \sigma(b, \xi)$

$\left(S_{W}(b, \xi), t\right) \overline{\left(S_{W}(b, \xi), f\right)} e^{\frac{-(t-\tau)^{2}}{2 \sigma(f)^{2}}} e^{\frac{-(f-\xi)^{2}}{2 \sigma^{2}}} d b d \xi$

By using uniform distributed property stated in [15] equation (7) can be modified as

$\left(S_{\sigma, W}, t, f\right)=\frac{1}{c_{w}} \int_{-\infty}^{\infty} \int_{-\infty}^{\infty} \sigma(b, \xi)\left(t, w_{S}^{b, \xi}\right)$

$\left(w_{S}^{b, \xi}, f\right) e^{\frac{-(t-b)^{2}}{2 \sigma^{2}}} e^{\frac{-(f-\xi)^{2}}{2 \sigma^{2}}} d b d \xi$

By using product theorem for Hilbert transform [16], localized operators in (8) can be turned as multi linear operators as follows

$$
\begin{aligned}
& \left(S_{\sigma, W}, t, f\right)=\frac{1}{c_{w}} \int_{-\infty}^{\infty} \int_{-\infty}^{\infty} \sigma(b, \xi)\left(w_{S}^{b, \xi}(t) * w_{S}^{b, \xi}(t-b)\right) \\
& \left(w_{S}^{b, \xi}(f) * w_{S}^{b, \xi}(f-\xi)\right) e^{\frac{-(t-b)^{2}}{2 \sigma^{2}}} e^{\frac{-(f-\xi)^{2}}{2 \sigma^{2}}} d b d \xi
\end{aligned}
$$


Where $\quad \sigma=$ Standard deviation.

$c_{w}=$ Normalized window coefficient.

$\xi=$ Scaling parameter on frequency axis.

$\mathrm{b}=$ Scaling parameter on time axis.

$*=$ convolution property

By winger transform [17], the window function can be localized in terms of signal complex conjugate model as follows

$\mathrm{w}(\mathrm{t}, \mathrm{f})=\frac{1}{\sqrt{2 \pi}} \int_{-\infty}^{\infty} x\left(t, b, \frac{\xi}{2}\right) x^{\prime}\left(f, b, \frac{\xi}{2}\right) d b d \xi ;$

The window width of the equation (10) can be considered as limitation since it will take into consideration the nature of analyzed signal. By including this window variation, finally the resultant multi localization operators obtained as

$\left(S_{\sigma, W}, t, f\right)=\frac{1}{c_{w}} \int_{-\infty}^{\infty} \int_{-\infty}^{\infty} \sigma(b, \xi) x\left(w_{S}^{b, \xi}(t) * w_{S}^{b, \xi}(t-\right.$

b)) $x^{\prime}\left(w_{S}^{b, \xi}(f) * w_{S}^{b, \xi}(f-\xi)\right) e^{\frac{-(t-b)^{2}}{2 \sigma^{2}}} e^{\frac{-(f-\xi)^{2}}{2 \sigma^{2}}} d b d \xi$;

By simplifying above equation finally

$$
\begin{aligned}
& \left(S_{\sigma, W}, t, f\right)= \\
& \frac{1}{c_{w \sqrt{2 \pi}}} \int_{-\infty}^{\infty} \int_{-\infty}^{\infty} \sigma(b, \xi) x_{w}\left(t, b, \frac{\xi}{2}\right) x^{\prime}{ }_{w}\left(f, b, \frac{\xi}{2}\right) e^{\frac{-(t-b)^{2}}{2 \sigma^{2}}} e^{\frac{-(f-\xi)^{2}}{2 \sigma^{2}}} d b d \xi
\end{aligned}
$$

The above mathematical formulation shows improved resolution consistency when compared with Standard S- Transform. It can be observed that window of the S-transform (12) accounts into the consideration of non stationary signal nature.

\section{Physical method for proposed algorithm}

In novel method of optimized frequency window proposed in $[12,13]$, a new differential frequency window is proposed in this section. The major aim of this window is to track all abrupt amplitude-frequency variations which exist in non stationary signal. One major problem during transform is whenever non stationary signal consists of abrupt amplitude variation equal to peak of guassian window at initial intervals of guassian function then some amplitude part of the non stationary signal is overlapped beyond the amplitude variations of guassian window. This makes some amplitude clipping during transform projection results in poor resolution. The same problem can also be observed at end intervals of guassian window. To avoid this problem a parameter ' $\beta$ ' is included in standard STransform which makes variation in guassian window shape. The proposed window major task is to maintain the neighboring frequencies of signal energy amplitudes should be projected to peak of guassian and the energy contribution of each component would not exceed its window duration.

Steps for variation of ' $\beta$ ':

a) Take initially $\beta_{\mathrm{t}}=\frac{\mathrm{k}}{\left(\mathrm{c}+\mathrm{a}\left(\Delta \mathrm{f}_{\mathrm{t}}\right)^{\frac{1}{2}}\right)}, \mathrm{k}>0$;

b) Assume window as normalized interval [0 1 1]

c) Perform Newton Raphson iteration $\beta_{t+1}=\beta_{t}-$ $\frac{w\left(\Delta \mathrm{f}_{\mathrm{t}}\right)}{w^{\prime}\left(\Delta \mathrm{f}_{\mathrm{t}}\right)}$;

d) Above iteration is repeated until

$$
\beta_{t+1} \cong \beta_{t}
$$

The window variations performed by Newton Raphson method [18] are taken from the section 2 (12) analytical identity which includes non linear variation of $\mathrm{x}(\tau)$. The term $\Delta \mathrm{f}_{\mathrm{t}}$ indicates the bandwidth of the guassian function and $\left(\Delta \mathrm{f}_{\mathrm{t}}\right)^{\frac{1}{2}}$ is fractional frequencies indulges in non linear periodic path of $x(\tau)$. The complete physical significance of $\left(\Delta f_{t}\right)^{\frac{1}{2}}$ can be observed in [19] as it involves complex nature during transformation. In this way entire aperiodic path of $\mathrm{x}(\tau)$ is traced with peak of guassian window until $\beta_{t+1} \cong \beta_{t}$.

Finally improved $S$ - Transform is given as

$S^{\prime}\left[t, f, \beta_{\mathrm{t}}\right]=\int_{-\infty}^{\infty} x(\tau) w\left(t-\tau, f, \beta_{t}\right) e^{-j 2 \pi f \tau} d \tau ;$

$S^{\prime}\left[t, f, \beta_{\mathrm{t}}\right]=\int_{-\infty}^{\infty} x(\tau) \frac{|f|}{\sqrt{2 \pi} \beta_{t}} e^{\frac{-\pi(t-\tau)^{2} f^{2}}{2 \beta_{t}^{2}}} e^{-j 2 \pi f \tau} d \tau ;$

Where $w\left(t-\tau, f, \beta_{t}\right)=\frac{|f|}{\sqrt{2 \pi} \beta_{t}} e^{\frac{-\pi(t-\tau)^{2} f^{2}}{2 \beta_{t}^{2}}}$;

$\beta_{\mathrm{t}}=\frac{\mathrm{k}}{\left(\mathrm{c}+\mathrm{a}\left(\Delta \mathrm{f}_{\mathrm{t}}\right)^{\frac{1}{2}}\right)}$;

$\left(\Delta \mathrm{f}_{\mathrm{t}}\right)^{\frac{1}{2}}=$ Fractional frequency value at different ' $\mathrm{t}$ ' intervals. $\mathrm{c}=\mathrm{Y}$ - axis intercept.

$\mathrm{a}=\mathrm{X}$ - axis intercept.

$\mathrm{k}=$ Normalized amplitude value.

By substituting $\beta_{\mathrm{n}}$ (13) in (17) the resultant improved $\mathrm{S}$ Transform is given as

$$
\begin{gathered}
S^{\prime}\left[t, f, \beta_{\mathrm{t}}\right]=\int_{-\infty}^{\infty} x(\tau) \frac{|f|}{\left.\sqrt{2 \pi(} \frac{\mathrm{k}}{\left(\mathrm{c}+\mathrm{a}\left(\Delta \mathrm{f}_{\mathrm{t}}\right)^{\frac{1}{2}}\right.}\right)} e^{\frac{-\pi(t-\tau)^{2} f^{2}}{2 \beta_{t}^{2}}} e^{-j 2 \pi f \tau} d \tau ; \\
\text { Or } \quad S^{\prime}\left[t, f, \beta_{\mathrm{t}}\right]=\int_{-\infty}^{\infty} x(\tau) \frac{|f|}{\sqrt{2 \pi k}}(\mathrm{c}+ \\
\left.\mathrm{a}\left(\Delta \mathrm{f}_{\mathrm{t}}\right)^{\frac{1}{2}}\right) e^{\frac{-\pi(t-\tau)^{2} f^{2}\left(\mathrm{c}+\mathrm{a}\left(\Delta \mathrm{f}_{\mathrm{t}}\right)^{\frac{1}{2}}\right)^{2}}{2 k^{2}}} e^{-j 2 \pi f \tau} d \tau
\end{gathered}
$$

The new window satisfies the normalization condition for the original S-transform window which ensures the invertibility of the modified S-transform given as

$$
\int_{-\infty}^{\infty} w\left(t-\tau, f, \beta_{\mathrm{t}}\right) d \tau=1
$$

Using the equivalent frequency domain definition of the Stockwell transform $[8,9]$, the improved discrete S-Transform is given as

$S^{\prime}\left[j T, \frac{p}{N T}, \beta_{\mathrm{nT}}\right]=\sum_{m=0}^{N-1} X\left[\frac{m+p}{N T}\right] e^{\frac{-\pi(n T)^{2} m^{2}\left(\mathrm{c}+\mathrm{a}\left(\Delta \mathrm{f}_{\mathrm{nT}}\right)^{\left.\frac{1}{2}\right)^{2}}\right.}{2 k^{2}}} e^{\frac{j 2 \pi m n}{N}} ;$

For $\mathrm{n} \neq 0$;

Where $\quad$ Input $=\mathrm{x}(\mathrm{m}) ; \mathrm{m}=0,1,2, \ldots ., \mathrm{N}-1$;

$\mathrm{H}$ (.) is the Discrete Fourier Transform (DFT) of $\mathrm{h}$ (.) defined for $\mathrm{p}=0,1,2 \ldots \mathrm{N}-1$;

Empirically the values of $\mathrm{c}, \mathrm{a}, \mathrm{n}$ and $\mathrm{k}$ will may not be adequate for some types of signals. It will be more appropriate to generate automatically adaptive parameters which respect the nature of analyzed signal. A Recursive Genetic Algorithm (RGA) [20] is used to select automatically the parameters c, a, n and k. Initially a test vector of values $\mathrm{c}, \mathrm{a}, \mathrm{n}$ and $\mathrm{k}$ are generated using Genetic algorithm. Where Genetic Algorithm (GA) is based on the mechanisms of natural selection and genetics has been developed since 1975 [20, 21]. RGA has been proven to be very efficient and stable in searching for global optimum solutions. Usually, a simple GA is mainly composed of three operations: selection, genetic operation, and replacement. A recursive fitness proportionate function is adapted for optimization [20, 21]. This fitness proportionate function will acts as feedback loop. The above process is repeated until achievement of optimized values $\mathrm{c}, \mathrm{a}, \mathrm{n}$ and $\mathrm{k}$ with respect to standard GA algorithm values.

The complete flow graph model of RGA is shown in Fig. 1. Optimized model. 


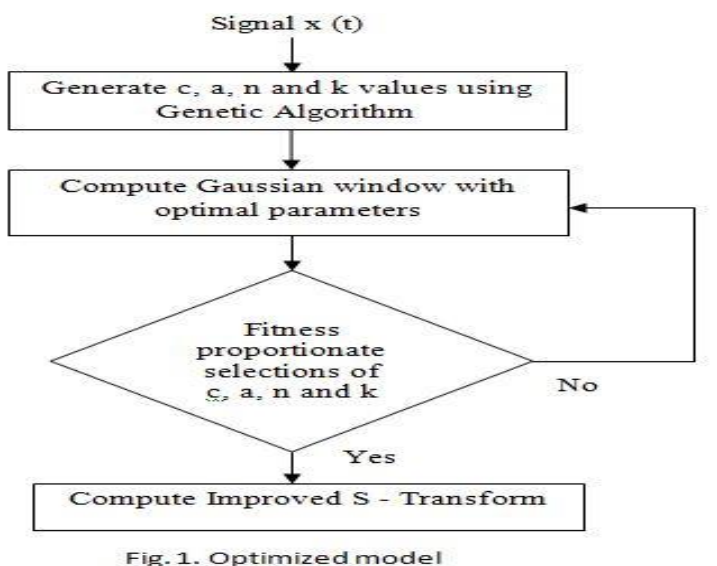

Finally as per in view of real time applications concerned, the proposed window transform is implemented on FPGA by using Xilinx 14.1 SPARTAN XC3S4000 [23]. The complete design flow of the FPGA implementations is as shown in Fig.2. Where input buffer consist of Non stationary and window transformed values. These values are transferred to the Xilinx Processor. The 12 bit output from processor is fed to DAC and output is viewed in CRO. For these transformation conversions CARDIAC algorithm is used for VHDL implementation [24].

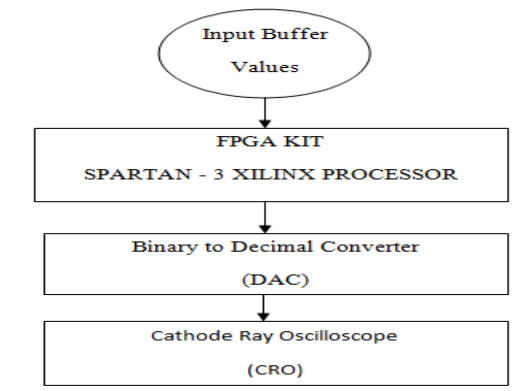

Fig .2. Design flow for FPGA implementation of proposed scheme

\section{Simulation results and discussions}

In this section, performance of proposed algorithm for the STransform is examined. Initially the proposed algorithm is tested for sample ECG data and compared with other TFRs: a) Short Time Fourier Transform (STFT), b) Wigner-Ville distribution (WVD) c) Standard S - Transform, d) Modified S Transform [12], e) Proposed S-Transform(22).

Test1:- Input: ECG data
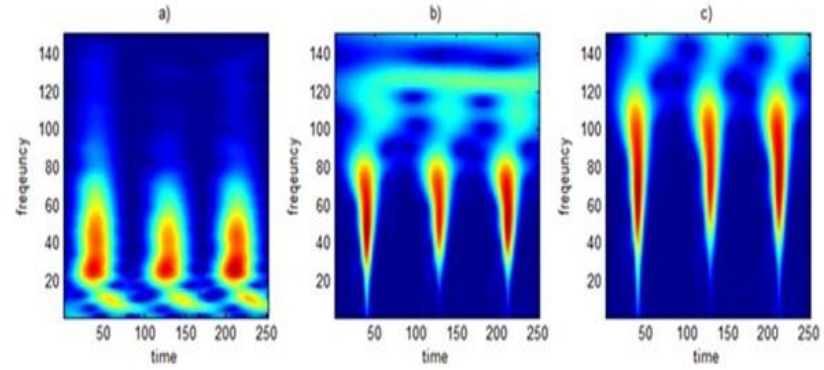

d)
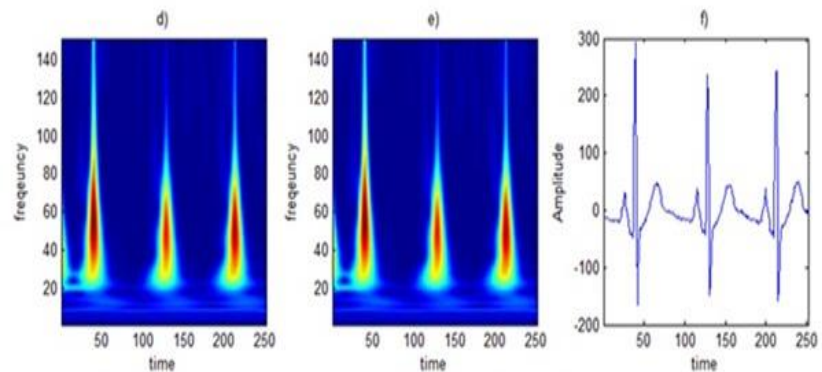

Fig. 3. a) Short Time Founer Transform(STFT), b) Wigner-Ville distribution (WVD) c) Standard STransform, d) Modified S - Transform [12], e) Proposed S-Transform(22).

From the results it can be concluded that TFR resolution of Modified S - Transform [12] and Proposed S-Transform are similar, better when compared with the STFT, WVD and Standard S-Transform. But the advantage of proposed STransform is when the signal is attacked suddenly with abrupt changes (abnormal changes) then the change of abnormality is traced faster when compared with other TFRs.

Test2:- An abrupt change of ECG data during transformation is considered and performed TFRs.

A particular concentration of Modified S-Transform [12] and Proposed S-Transform (22) has been evaluated with different elapsed time delay intervals.

i) Case at $\tau=0.521 \mathrm{~ms}$
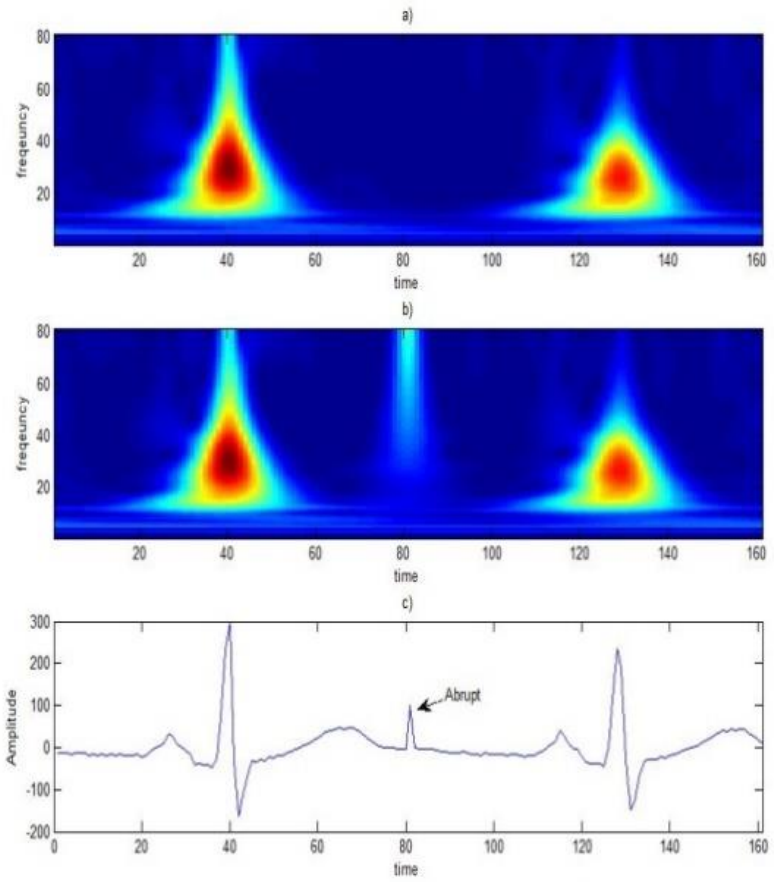

Fig 4. a) Modfied Stranstorm[12], b) Proposed Stranstom (22), c) ECG data

From Fig. 4. It can be observed that small sign of indication for abrupt variation in proposed S-Transform (22) but it was not found in Modified S-Transform [12].

ii) Case at $\tau=1.054 \mathrm{~ms}$ 

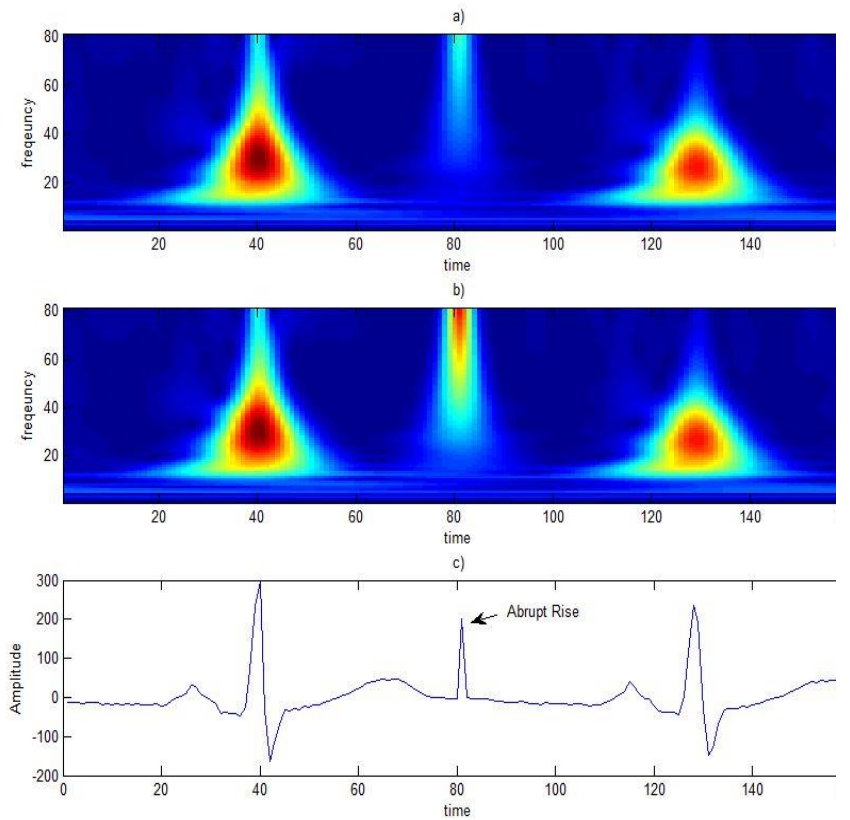

Fig.5. a) Modified S-transform[12], b) Proposed S-transform(22), c)ECG data

From Fig. 5. It can be observed that proposed S-Transform (22) gives better localization when compared with Modified STransform [12].

iii) Case at $\tau=1.640 \mathrm{~ms}$
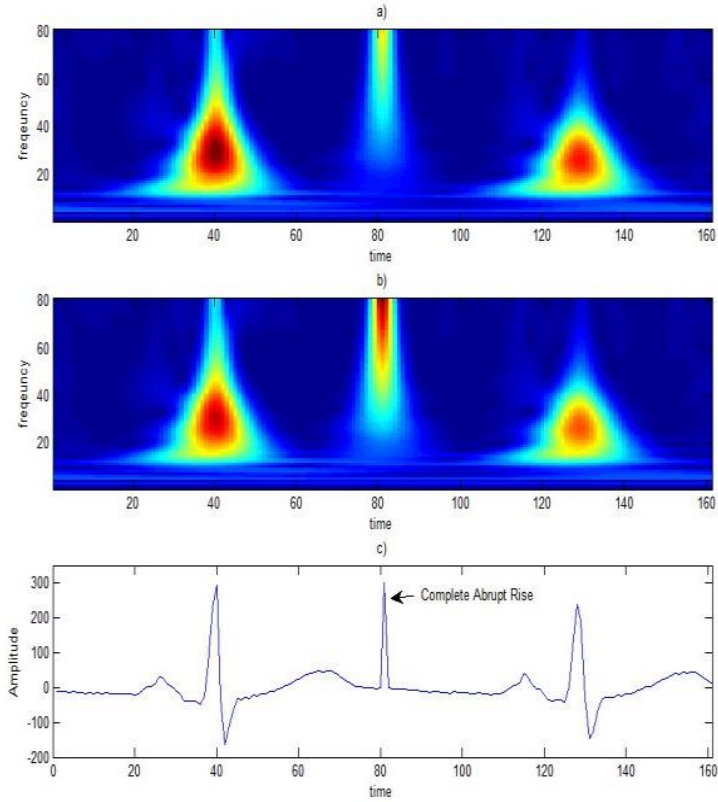

Fig.6. a) Modfied S.transform[12], b) Proposed S.transform(22), c|ECG data

Fig.6. Result shows that proposed algorithm (22) gives better resolution for abrupt changes in estimated instantaneous amplitude-instantaneous frequency (IF) values [10].

A sample variation of Guassian window with respect to ' $\beta_{\mathrm{n}}$ ' during transform is shown in Fig. 7.

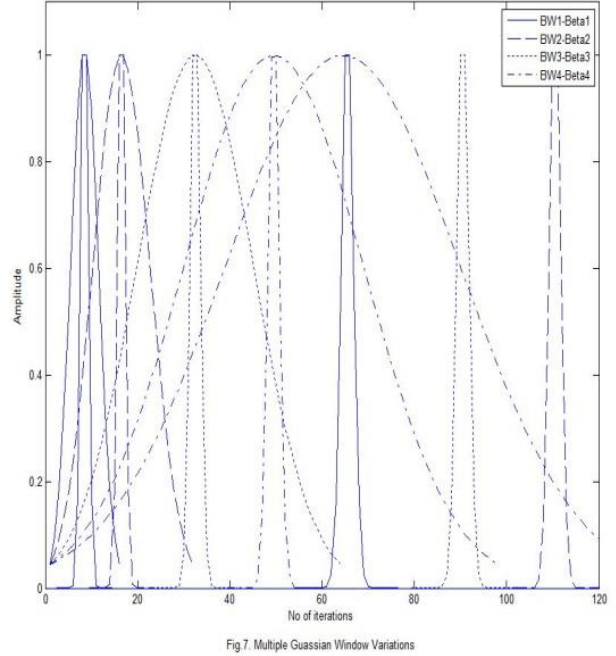

Furthermore the time delay taken for complete transformation of proposed algorithm is less when compared with other TFRs. Table. 1. Shows Concentric Metric Measures (CMM) of proposed algorithm with other TFRs.

Table. 1. CMM comparison of different TFRs

\begin{tabular}{|c|c|c|c|c|c|}
\hline CMM & STFT & WVD & $\begin{array}{l}\text { S- } \\
\text { Trans- } \\
\text { form }\end{array}$ & $\begin{array}{l}\text { Modi- } \\
\text { fied } \\
\text { S- } \\
\text { Trans- } \\
\text { form } \\
{[18]}\end{array}$ & $\begin{array}{l}\text { Pro- } \\
\text { posed } \\
\text { S- } \\
\text { Trans- } \\
\text { form }\end{array}$ \\
\hline $\begin{array}{l}\text { Abrupt peak- } \\
\text { Pixel Resolu- } \\
\text { tion (Mean) }\end{array}$ & 0.5254 & 0.5145 & 0.3645 & 0.2232 & 0.2012 \\
\hline $\begin{array}{l}\text { Abrupt peak- } \\
\text { Pixel Resolu- } \\
\text { tion (Standard } \\
\text { Deviation) }\end{array}$ & 0.4213 & 0.3945 & 0.2554 & 0.1942 & 0.1524 \\
\hline Delay (ms) & 2.284 & 2.021 & 1.912 & 1.642 & 1.562 \\
\hline
\end{tabular}

Finally proposed algorithm is examined in presence of noise. Accuracy of the instantaneous frequency estimation based on peak values is observed [10]. A sample of ECG data [29] is taken and contaminated with additive white guassian noise (AWGN) and performed signal to noise ratio (SNR) versus Mean Square Error (MSE).

Where

$\mathrm{SNR}=10 \log _{10}\left(\frac{A^{2}}{\sigma_{n}^{2}}\right)$;

$\sigma_{n}^{2}=$ Variance of the noise.

$\mathrm{A}=$ Signal amplitude (Normalized value ' 1 ').

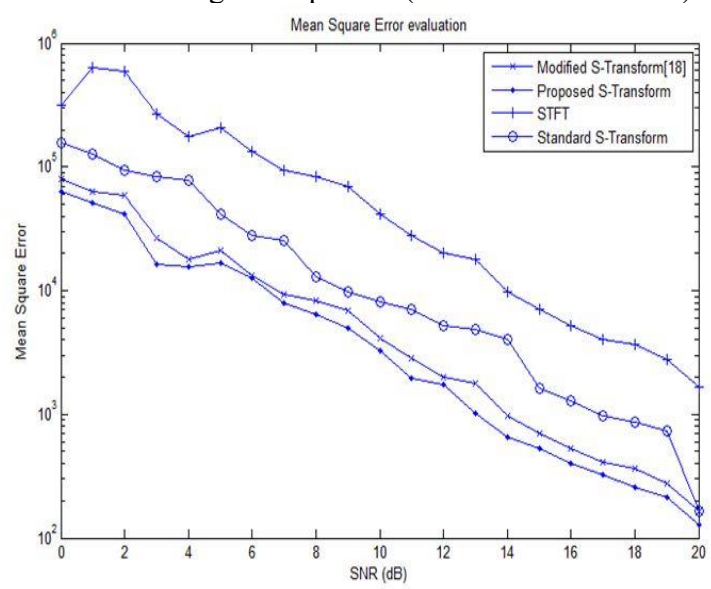

Fig. 8. MSE error comparison with different TFRs

From Fig. 8 It can be observed that proposed algorithm yields less MSE when compared with other TFRs. 
FPGA results for proposed technique:

The sample values of input signal X(nT) and window coefficients $\mathrm{W}(\mathrm{nT})$ are processed to processor by buffer system. The resultant output values are in the form of binary (12-bit) data. In order to observe de noising output in CRO these values are given to DAC. Fig. 9 shows the RTL schematic of FPGA implementation.

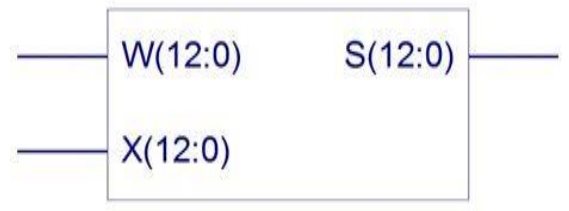

\section{Fig.9. RTL Schematic for proposed transform}

Fig. 10. a) Shows a signal containing without any noise. Then corresponding transient signal added with white Gaussian noise consists SNR of $10 \mathrm{~dB}$ is shown in Fig. 10. b). Finally filtered output is shown in Fig. 10. c).
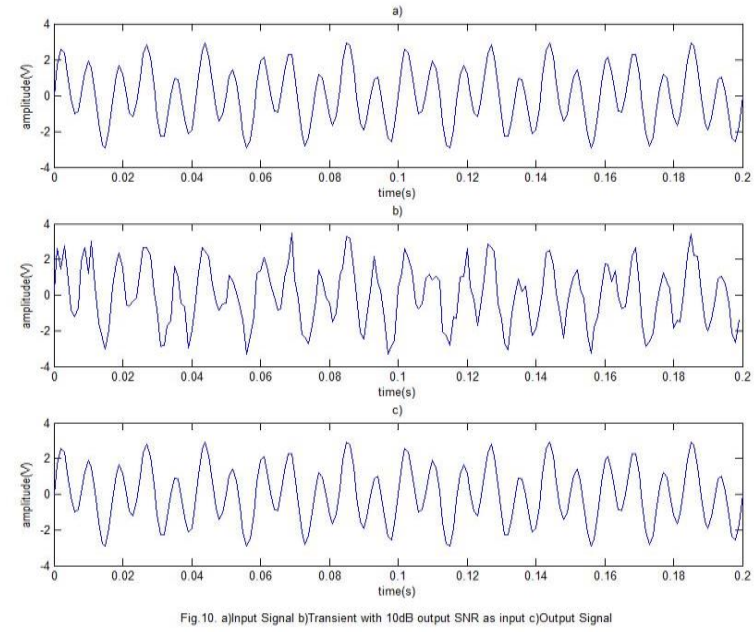

\section{Conclusion}

In this paper, a differential frequency window S-Transform is proposed. The proposed algorithm is evaluated with other TFRs. Under normal environment conditions proposed algorithm TFR is equivalent to modified S-Transform [12]. But the proposed algorithm gives improved results in abrupt environment when compared with other TFRs. An attempt of non stationary signal behavior is considered by proposed window with localization parameters. Moreover it was observed that proposed method produces less MSE when compared with other TFRs. But the major limitation of proposed method is when $\beta_{n}$ goes for higher order Newton Raphson method then delay factor increases enormously.

\section{Acknowledgements}

The author wishes to thank KLEF (Research \& Development Centre) for providing financial support to carry research work.

\section{References}

[1] CRAMÉR H., "On some classes of non stationary stochastic processes", Proc. $4^{\text {th }}$ Berkeley Sympos. Math. Stat. Prob., University of California Press, pp. 57-78, 1961.

[2] B. Boashash, Time-Frequency Signal Analysis and Processing: A Comprehensive Reference, Elsevier, Amsterdam; Boston, 2003.

[3] Cohen L. Time-frequency analysis. Englewood Cliffs, NJ: Prentice-Hall, PTR; 1995.
[4] Tolimieri, Richard, and Myoung An, eds. Time-frequency representations. Springer, 1998.

[5] Stockwell, Robert Glenn. "A basis for efficient representation of the S-transform." Digital Signal Processing 17.1 (2007): 371-393

[6] Goswami, Jaideva C., and Andrew K. Chan. Fundamentals of wavelets: theory, algorithms, and applications. Vol. 233. John Wiley \& Sons, 2011.

[7] Mallat, Stéphane. A wavelet tour of signal processing. Academic press, 1999.

[8] Stockwell, Robert Glenn, Lalu Mansinha, and R. P. Lowe. "Localization of the complex spectrum: the S transform." Signal Processing, IEEE Transactions on44.4 (1996): 998-1001.

[9] STOCKWELL R.G., Why use the S transform?, in PseudoDifferential Operators: Partial Differential Equations and Time-Frequency Analysis, Editors: L. Rodino, B.-W. Schulze and M.W.Wong, Fields Institute Communications Series 52, American Mathematical Society, 2007, 279-309.

[10] Boashash B. Estimating and interpreting the instantaneous frequency of a signal-part 1: fundamentals. Proc IEEE 1992; 80(4):520-38.

[11] Dash, P. K., K. B. Panigrahi, and G. Panda. "Power quality analysis using S-transform." Power Delivery, IEEE Transactions on 18.2 (2003): 406-411.

[12] Assous and Boashash: Evaluation of the modified S transform for time-frequency synchrony analysis and source localisation. EURASIP Journal on Advances in Signal Processing 2012 2012:49.

[13] Djurović, Igor, Ervin Sejdić, and Jin Jiang. "Frequencybased window width optimization for S-transform." AEUInternational Journal of Electronics and Communications 62.4 (2008): 245-250.

[14] WONG M.W., Wavelet Transforms and Localization Operators, Operator Theory: Advances and Applications 136 Birkh"auser, 2002.

[15] Case, William B. "Wigner functions and Weyl transforms for pedestrians."American Journal of Physics 76.10 (2008) 937-946.

[16] Bedrosian, E. (December 1962), "A Product Theorem for Hilbert Transforms", Rand Corporation Memorandum (RM3439-PR)

[17] B. Boashash, P. O'Shea, Polynomial Wigner-Ville distributions and their relationship to time varying higher order spectra, IEEE Transactions on Signal Processing 42(1994)216-220.

[18] Ypma, Tjalling J. "Historical development of the NewtonRaphson method."SIAM review 37.4 (1995): 531-551.

[19] Deekshitulu, G. V. S. R., and J. Jagan Mohan. "Fractional difference inequalities." Communications in Applied Analysis 14.1 (2010): 89

[20] Georgilakis, P. S. "Recursive genetic algorithm-finite element method technique for the solution of transformer manufacturing cost minimisation problem." iet electric power applications 3.6 (2009): 514-519.

[21] Davis, Lawrence, ed. Handbook of genetic algorithms. Vol. 115. New York: Van Nostrand Reinhold, 1991.

[22] Moukadem, Ali, et al. "Stockwell transform optimization applied on the detection of split in heart sounds." Signal Processing Conference (EUSIPCO), 2013 Proceedings of the 22nd European. IEEE, 2014.

[23] Ramachandran, Seetharaman. Digital VLSI Systems Design: A Design Manual for Implementation of Projects on FPGAs and ASICs Using Verilog. Springer Science \& Business Media, 2007.

[24] Meyer-Bäse, Uwe, Anke Meyer-Bäse, and Wolfgang Hilberg. "Coordinate rotation digital computer (CORDIC) synthesis for FPGA." Field-Programmable Logic Architectures, Synthesis and Applications. Springer Berlin Heidelberg, 1994. 397-408 